\title{
Level of muscle regeneration in limb-girdle muscular dystrophy type 2 I relates to genotype and clinical severity
}

Thomas O Krag ${ }^{1 *}$, Simon Hauerslev ${ }^{1}$, Marie Louise Sveen ${ }^{1}$, Malene Schwartz ${ }^{2}$ and John Vissing ${ }^{1}$

\begin{abstract}
Background: The balance between muscle regeneration and ongoing degeneration is a relationship that greatly influences the progression of muscular dystrophy. Numerous factors may influence the muscle regeneration, but more information about the relationship between genotype, clinical severity and the ability to regenerate is needed.

Methods: Muscle biopsies were obtained from the tibialis anterior muscle, and frozen sections were stained for general histopathological and immunohistological evaluation. Differences between groups were considered statistical significant at $P<0.05$ using Student's unpaired $t$-test.

Results: We found that all patients with limb-girdle muscular dystrophy type 2l (LGMD2I) had a large number of internally nucleated fibers, a sign of previous regeneration. The level of expression of muscle-specific developmental proteins, such as neonatal myosin heavy chain (nMHC) and myogenin, was related to the clinical severity. Additionally, we found that the majority of nMHC-positive fibers did not stain positively for utrophin in patients who were compound heterozygous for the L276I mutation, suggesting that the predominant form of regeneration in these patients is fiber repair rather than formation of new fibers. Double staining showed that many smaller nMHC-positive fibers were positive for antibodies against the glycosylation on $\alpha$-dystroglycan, suggesting that such glycosylation may be a result of muscle regeneration.

Conclusion: Severely affected patients with LGMD2I have a high level of muscle degeneration, which leads to a high rate of regeneration, but this is insufficient to change the imbalance between degeneration and regeneration, ultimately leading to progressive muscle wasting. Detailed information regarding the level and rate of muscle regeneration and potential obstructions of the regenerative pathway should be of use for future therapies involving satellite-cell activation.
\end{abstract}

\section{Background}

Limb-girdle muscular dystrophy type 2I (LGMD2I) was molecularly characterized in 2001 as caused by mutations in the gene for fukutin-related protein (FKRP) [1]. It has been suggested that FKRP is involved in glycosylation of $\alpha$-dystroglycan ( $\alpha$-DG) [2], a protein that stabilizes the link between the contractile apparatus and the extracellular matrix (ECM) [3]. Although it is believed to possess enzymatic properties, the exact function of FKRP has not yet been elucidated. It has been suggested

\footnotetext{
* Correspondence: thomas.krag@rh.regionh.dk

${ }^{1}$ Neuromuscular Research Unit, Department of Neurology Rigshospitalet, University of Copenhagen, Denmark Full list of author information is available at the end of the article
}

that mutations affecting the translocation of FKRP from the endoplasmic reticulum to the Golgi apparatus invariably lead to severe phenotypes, whereas mutations that do not affect lodging of FKRP in the Golgi membrane may lead to moderate phenotypes [4]. However, other investigators have suggested that the main pathogenic mechanism relates to a lack of interaction between FKRP and its substrate(s) in the Golgi [5].

In Denmark, LGMD2I accounts for $40 \%$ of the total number of recessively inherited cases of LGMD2 [6]. Most patients with LGMD2I are homozygous for the mutation L276I (826A $>C$ transversion). This genotype is generally associated with a mild phenotype, whereas
C Biomed Central 
patients who are compound heterozygous for this mutation generally display a more severe phenotype $[7,8]$.

There is no known substitute for FKRP, and loss of its function in LGMD2I leads to degeneration of myofibers and secondary loss of proteins that are important for structure and function, such as $\alpha-\mathrm{DG}$ and merosin $[9,10]$. To counterbalance degeneration, a muscle must regenerate its fibers, and establishing the regenerative response to muscle damage in muscular dystrophies may provide important clues to therapeutic interventions.

In general, muscle damage leads to activation of quiescent satellite cells residing between the sarcolemma and basal lamina. Once the satellite cell has fused with the myofiber, its nucleus migrates to the site that requires repair. This migration is visible histologically as internally nucleated fibers, and counting these has traditionally been one way of determining muscle regeneration [11]. However, internally nucleated fibers may not fully explain the temporal pattern of a regenerative response to ongoing degeneration, because internal nuclei can reside in the cytoplasm of myofibers for several months.

In this study, we determined the temporal regenerative response to muscle degeneration in patients with LGMD2I, using developmental and myogenic markers that are only expressed for days or weeks, such as MyoD (0 to 2 days) and myogenin (1 to 7 days), neonatal myosin heavy chain (nMHC, 1 to 3 weeks) and utrophin (weeks to months) [12-14]. We assessed the recent regeneration in muscle fibers of patients homozygous and compound heterozygous for the L276I mutation, and correlated this with the age at biopsy and duration of LGMD2I in patients, as the capacity to regenerate normally decreases with age [15].

\section{Methods}

The study was approved by the Scientific Ethics Committee of Copenhagen and Frederiksberg Counties approved this study, and all patients provided informed consent.

\section{Patients}

In total, 22 patients with LGMD2I participated in the study (Table 1). Clinical onset was defined as the first time patients noticed symptoms such as decreased walking distance, muscle pain after physical exertion, trouble walking up stairs, rising from a seated or crouched position, and tendency to fall. Of the 22 patients with LGMD2I, 17 (eight male, nine female; mean \pm SD age $44 \pm 14$ years, range 23 to 65 years) were homozygous for the L276I mutation and had disease onset at $19 \pm 11$ years of age (range 2 to 36 years). Six of these patients had disease onset in childhood, and the rest had a later onset. Duration of illness was defined as the period from age at onset to age when the biopsy was taken. The remaining five patients (one male, four female; mean \pm SD age $27 \pm 12$ years, range 13 to 40 years,) were heterozygous for the L276I mutation, and their disease onset occurred at $6 \pm 4$ years of age (range 3 to 12 years). Muscle morphology was evaluated in samples from the tibialis anterior. The strength of ankle dorsal flexion was therefore tested by dynamometry and used as a measure of clinical severity for the biopsied muscle [16]; it was normalized to the ankle dorsal flexion (196 $\pm 52 \mathrm{~N}$ ) determined in healthy subjects (14 male, 13 female; age $32 \pm 12$ years, range 16 to 59 years). From this group, six healthy controls (three female, three male; age $45 \pm 11$ years, range 31 to 55 years), formed the control group for all histology and immunohistology in the study.

\section{Muscle biopsy}

Muscle biopsies were obtained from the tibialis anterior muscle by percutaneous needle biopsy technique using a 5-mm Bergstrøm needle. Biopsies were snap-frozen in isopentane, cooled in liquid nitrogen, and stored at $-80^{\circ}$ $\mathrm{C}$ before being cut into sections on a cryostat.

\section{Histology and immunohistochemistry}

Sections 10 to $12 \mu \mathrm{m}$ thick were stained with hematoxylin and eosin (H\&E) for general histopathological evaluation and assessment of internal nuclei, defined as non-peripheral internal nuclei. Full sections were evaluated for the percentage of internal nuclei, necrotic and ring fibers, and the level of fibrosis and muscle-fat infiltration. A myofiber was considered internally nucleated

Table 1 Clinical and genetic presentation of patients with limb-girdle muscular dystrophy type 21

\begin{tabular}{|c|c|c|c|c|c|}
\hline Mutation & Gender, M:F & Age at biopsy, years & Onset of disease ${ }^{a}$, years & Lost ambulation & Respiratory aid \\
\hline L276I/L276I & $9: 8$ & $44 \pm 14$ & $19 \pm 11$ & 3 patients & No \\
\hline L276I/Y307N & M & 35 & 7 & Yes & $\mathrm{BiPAP}^{\mathrm{b}}$ \\
\hline L276I/L202Q & $\mathrm{F}$ & 40 & 4 & Yes & Respirator \\
\hline L276I/A157P & $\mathrm{F}$ & 16 & 3 & No & No \\
\hline L276I/P462S & $\mathrm{F}$ & 31 & 12 & Yes & No \\
\hline L276I/Y307N & $\mathrm{F}$ & 13 & 3 & Yes & BiPAP \\
\hline
\end{tabular}

${ }^{\mathrm{a} A g e}$ when patient first reported symptoms.

${ }^{\mathrm{a}}$ Bilevel positive air pressure. 
if at least one nucleus was non-peripheral. A three-point visual analog score was used to assess pathological severity as described previously by Fanin et al. (1 = active dystrophic process (marked increase in fiber-size variability, active degeneration and regeneration, marked increase in connective tissue); 2 = moderate dystrophic process (marked increase in fiber-size variability, increased internal nuclei, few degenerating and regenerating fibers, slight increase in connective tissue); and $3=$ mild myopathic picture (moderate increase in fiber-size variability, increase in internal nuclei)) [17]. Two investigators ( $\mathrm{SH}$ and $\mathrm{TOK}$ ), who were blinded to genotype, assessed all biopsies.

For immunohistochemistry, all sections were fixed in a 1:1 mixture of acetone and methanol or in $4 \%$ paraformaldehyde (for MyoD), and were subsequently blocked using 3\% FCS in PBS, then stained. Primary antibodies were diluted in blocking buffer (1:100). To assess the number of myofibers presently undergoing regeneration, sections were double-stained with antibodies to $\mathrm{nMHC}$ (Vector Laboratories, Burlingame, CA, USA) and utrophin (Santa Cruz Biotechnology, Santa Cruz, CA, USA). Activated satellite cells were visualized with MyoD antibodies (Vector Laboratories), and differentiated satellite cells (myoblasts) were visualized with myogenin antibodies (clone F5D, developed by Dr Woodring E. Wright, Developmental Studies Hybridoma Bank, under the auspices of the NICHD and maintained by The University of Iowa, Iowa City, IA, USA). Size and shape was used as criteria to separate myogenin-positive atrophic fibers from myogenin-positive regenerating fibers. All positive fibers were counted, and calculated as a percentage of the total number of fibers. To determine the effect of loss of function of FKRP, we assessed the presence of glycosylated $\alpha$-DG (clone VIA4-1, Upstate, Charlottesville, VA, USA), following the manufacturer's protocol. Alexa 488 and Alexa 594 (Invitrogen, Carlsbad, CA, USA) secondary anti-mouse and anti-goat antibodies were used at 1:500 dilution in blocking buffer. A microscope with epifluorescence was used for all microscopy (80i; Nikon, Tokyo, Japan), and pictures were taken using a $10 \times$ objective. Pictures were merged for quantification of the entire section.

\section{Statistical analysis}

Statistical significance between groups was determined using Student's unpaired $t$-test and $P<0.05$ was considered significant. All numbers provided are mean \pm SD. Linear regression analysis was performed for internal nuclei versus age (seventeen L276I homozygous patients and five heterozygous patients) and non-linear regression analysis for $\mathrm{nMHC}$ versus age and duration of illness (twelve L276I homozygous patients and five compound heterozygous patients; biopsies from five homozygous patients were not available for immunohistochemical staining, as only H\&E-stained sections were available). $P$-values were determined from a Pearson correlation coefficient table of critical values.

\section{Results \\ Morphology}

The muscle-biopsy sections from the 22 patients had an average of $729 \pm 409$ myofibers, whereas those from the six healthy subjects had $1351 \pm 400$. We found a fourfold lower $(P<0.00002)$ normalized ankle dorsal flexor force for patients who were compound heterozygous compared with those homozygous for the L276I mutation (Figure 1A). Patients homozygous for the L276I mutation had a rather varied pattern of glycosylated $\alpha$ dystroglycan staining in the sarcolemma, ranging from most fibers being positive, to an interspersed pattern with half the fibers positive and half negative, to very few positive fibers (Figure 1B). In patients who were compound heterozygous for the L276I mutation, glycosylated $\alpha$-dystroglycan was seen in less than $20 \%$ of fibers, with ring fibers staining strongly (Figure 1B). Compared with healthy normal muscle, H\&E-stained muscle sections from homozygous patients revealed a mild to severe pathology, whereas the muscle morphology of compound heterozygous patients invariably showed severe pathology (Figure 1C). Pathological severity was significantly different between groups (Figure 2A), but there were no significant differences in numbers of necrotic fibers, ring fibers or internally nucleated fibers between the homozygous and compound heterozygous patients (Figure 2B-D). Fiber splitting was seen in three of the homozygous patients and in all of the compound heterozygous patients. Healthy subjects did not display any abnormal morphology.

\section{Regeneration}

To assess the level of recent regeneration (within the previous 1 to 4 weeks), we stained for nMHC and utrophin. We noted that only small, newly regenerating myofibers stained positive for both nMHC and utrophin, whereas mature fibers with internal nuclei stained positive for nMHC only (Figure 3A-B). The number of small, newly regenerating fibers positive for both $\mathrm{nMHC}$ and utrophin did not differ significantly between the groups (Figure 3C). By contrast, the patients who were compound heterozygous for L276I had approximately seven times more nMHC-positive/utrophin-negative myofibers than did homozygous patients (Figure 3D, $P<$ 0.03). A significant difference in $\mathrm{nMHC}$ expression between homozygous and compound heterozygous patients was seen (Figure 3E, F). To confirm this finding, we stained for the transcription factors that initiate the myogenic program in satellite cells. Although there 


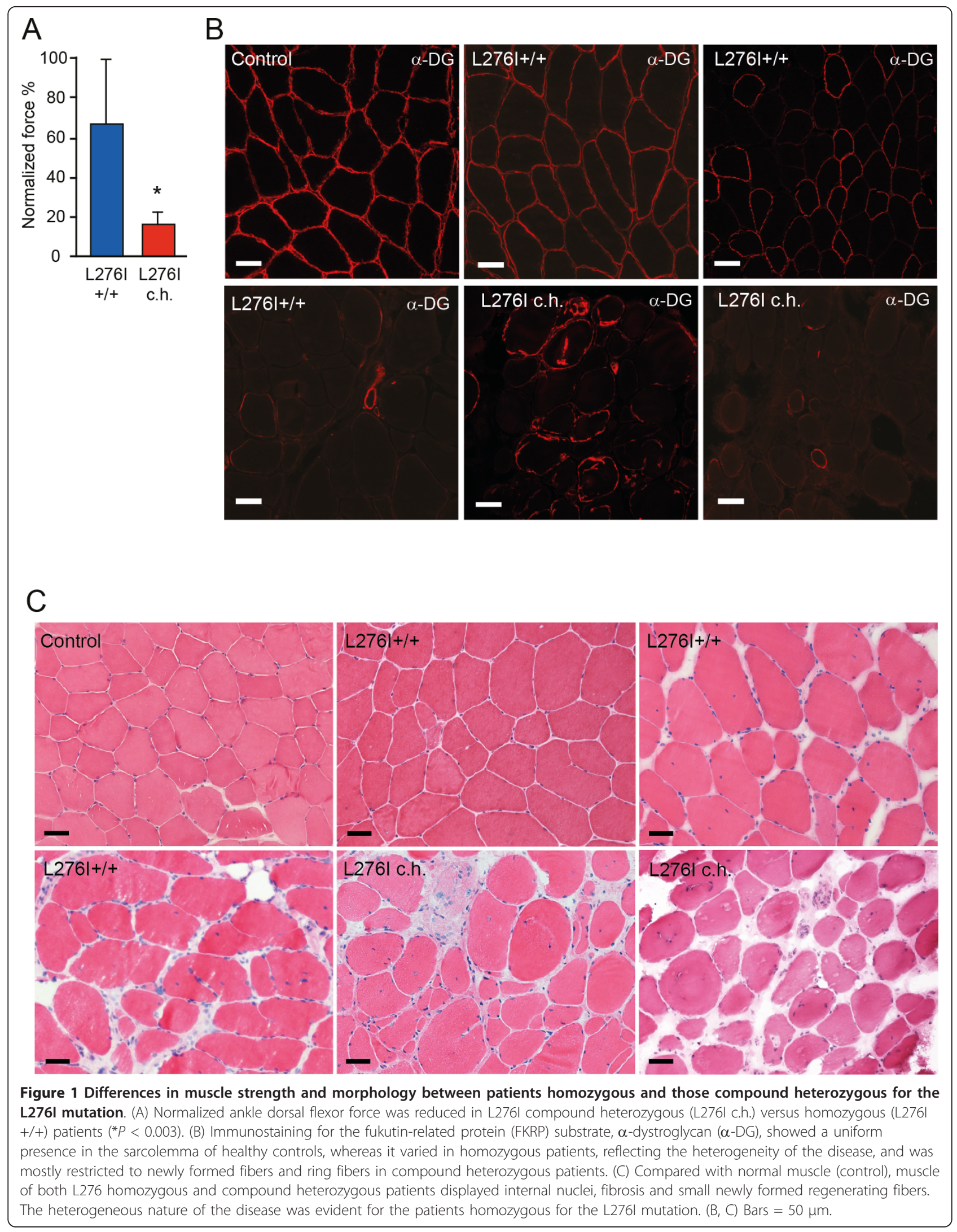



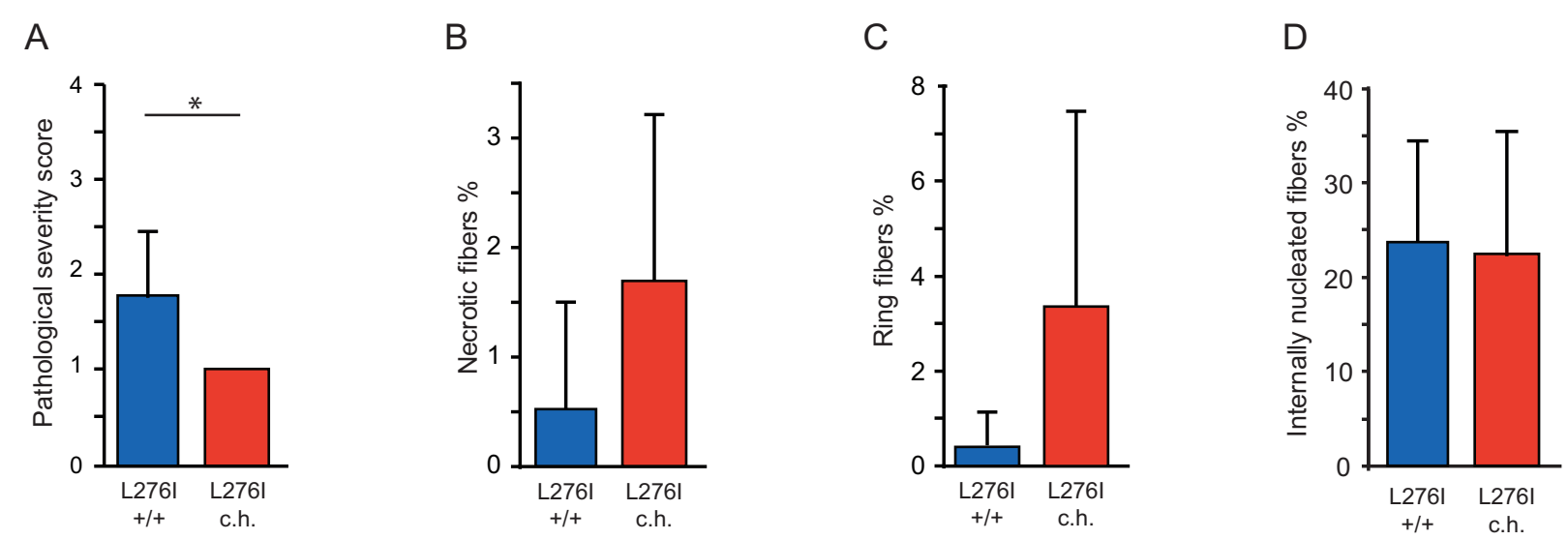

Figure 2 Histopathological evaluation of patients with LGMD2I. (A) The pathological severity score $(1=$ extensive degeneration of the muscle, 2 = moderate pathological changes in morphology, 3 = mild morphological changes), reflecting the overall morphology of the muscle in terms of degeneration, regeneration, and extent of fatty fibrous-tissue infiltration, shows that there was a significant difference between the group of patients with LGMD2I homozygous (blue bar) and the group compound heterozygous (red bar) for the L276I mutation. (B-D) There was no significant difference in number of (B) necrotic fibers, (C) ring fibers or (D) internally nucleated fibers between patients homozygous (blue bar) and those compound heterozygous (red bar) for the L276I mutation. ${ }^{*} P<0.05$.

were no MyoD-positive nuclei (data not shown), we noted that the number of myogenin-positive nuclei in the compound heterozygous patients trended towards an increase $(P<0.12)$, consistent with the nMHC finding (Figure 3G). Neither marker was found in any of the healthy subjects.

Dual labeling with nMHC and glycosylated $\alpha$-dystroglycan using the VIA4-1 antibody showed that the majority of small newly formed fibers staining positive for $\mathrm{nMHC}$ also stained positive for $\alpha$-dystroglycan. There was an almost inverse staining pattern in groups of regenerating fibers (Figure 4A-C).

We found no relationship between regeneration in terms of internally nucleated fibers and clinical severity and aging (data not shown). However, there was a significant relationship $(P<0.005$ for all patients) between nMHC-positive fibers and clinical severity in terms of ankle dorsal flexion (Figure 5A) and duration of disease $(P<0.05$, Figure $5 \mathrm{~B})$. In addition, we noticed a tendency for $\mathrm{nMHC}$-positive fibers to decline with age in the L2761 homozygous patients $(P<0.052$, data not shown). Immunohistochemistry was carried out on biopsies from twelve homozygous and five compound heterozygous patients.

\section{Discussion}

Most reports on LGMD2I have dealt with the clinical presentation and genetics of the disease, but little is known about the muscle morphology and regeneration of this disorder. In the absence of a substitute for FKRP, muscle fibers must rely on regeneration to counterbalance ongoing degeneration. In the present study, we determined the extent of this regeneration and whether the regenerative response is related to the clinical severity of LGMD2I, using some of the most commonly used markers for regeneration.

The key findings of the present study are that the level of early regeneration, as indicated by $\mathrm{nMHC}$ expression, was higher in patients who were compound heterozygous than in those homozygous for the L276I mutation, and that this regeneration was related to clinical severity. In addition, early regeneration tended to decrease with the duration of illness. Nevertheless, the apparent higher level of regeneration in compound heterozygous patients seems to be insufficient to maintain muscle mass, because the muscle wasting progresses more rapidly than in homozygous patients. The more severe phenotype in compound heterozygous patients was accompanied by a severe depletion of glycosylated $\alpha-$ dystroglycan. In these patients, $\alpha$-dystroglycan staining was generally restricted to small regenerating fibers and large ring fibers, which is possibly caused by a failed regenerative process after contractures [18]. This expression is consistent with previous findings that dystroglycan is upregulated during regeneration [19]. In this study, the sometimes inverse staining pattern we saw with $\mathrm{MHC}$ and $\alpha$-dystroglycan double-labeling suggests that as nMHC expression is reducing, the level of glycosylated $\alpha$-dystroglycan is increasing. Small regenerating fibers are believed to have added membrane stability because of increased utrophin and $\alpha-7$ integrin, hence the glycosylated $\alpha$-dystroglycan may persist for a longer period in these fibers compared with mature fibers $[20,21]$. Ultimately, as the protective higher level of proteins important for regeneration starts to decline, the glycosylated $\alpha$-dystroglycan presumably disappears. In 


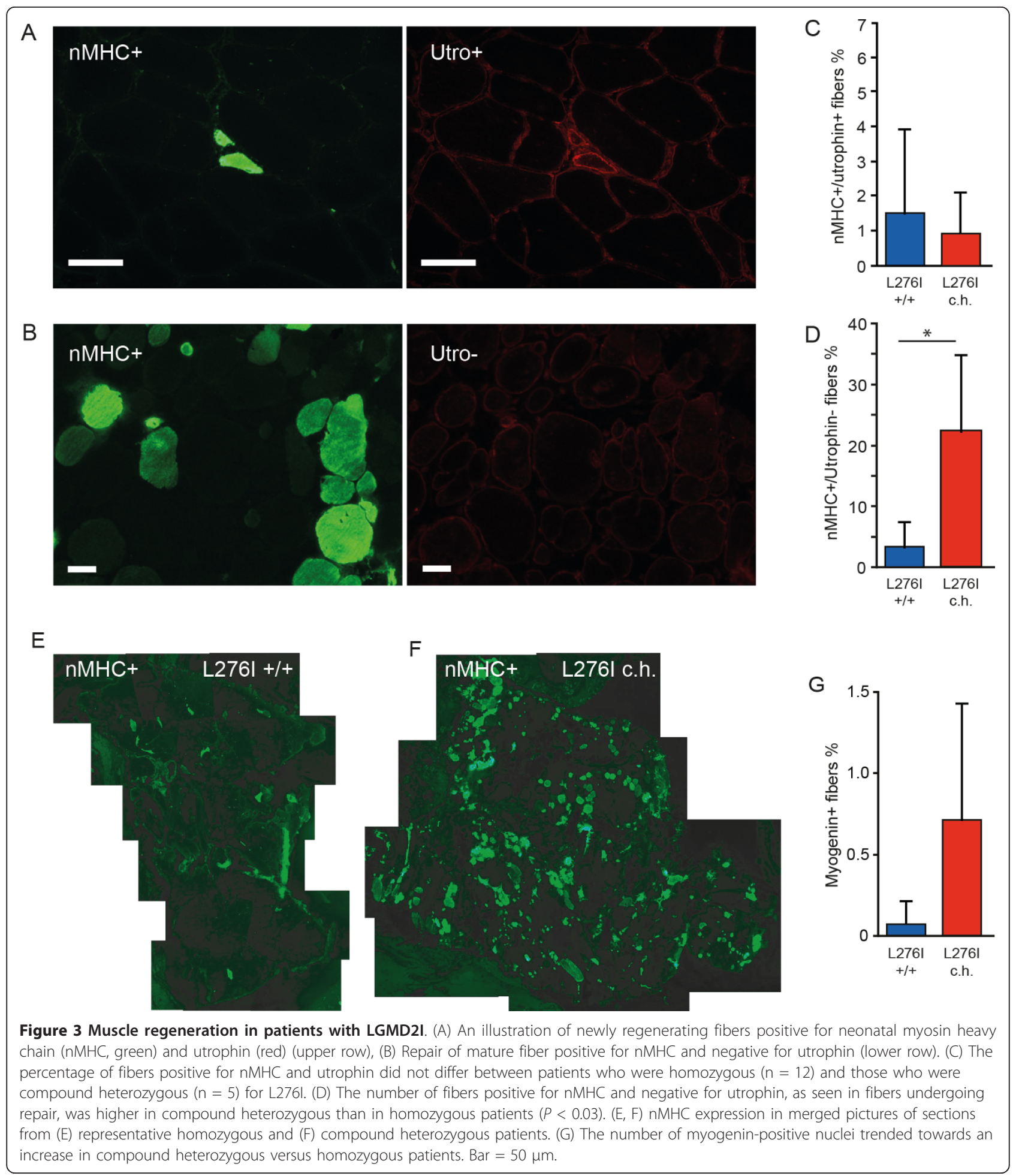

Duchenne muscular dystrophy (DMD), utrophin, an autolog of dystrophin, can to some extent substitute for the missing dystrophin [22,23]. It is not known whether a functional substitute for FKRP that could delay the progression of LGMD2I exists, or whether all glycosylated $\alpha$-dystroglycan originates from what basic functionality the mutated FKRP retains.

Although the L276I mutation affects FKRP, it retains some basic functionality, as suggested by the $\alpha$-dystroglycan staining in homozygous patients. Carriers of 

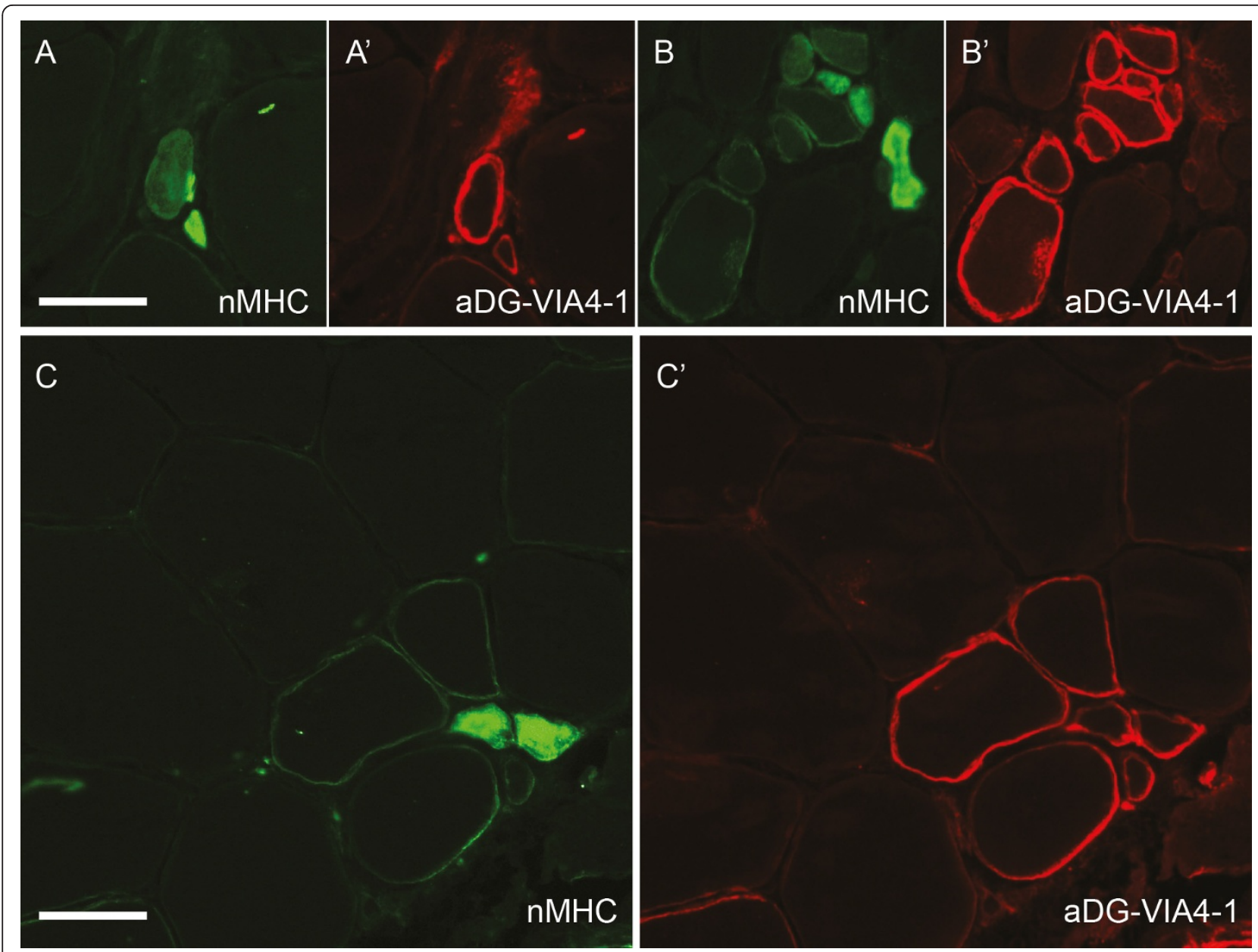

Figure 4 Co-expression of neonatal myosin heavy chain (nMHC) and $\boldsymbol{\alpha}$-dystroglycan. Many nMHC-positive fibers (A-C) also expressed $\alpha$ dystroglycan $\left(A^{\prime}-C^{\prime}\right)$. An inverse staining pattern was visible in small $n M H C$-positive fibers. (A) As the level of nMHC decreased, ( $\left.A^{\prime}\right) \alpha-$

dystroglycan increased. In a group of regenerating fibers, (B) the level of nMHC was low in all but two fibers whereas (B') all but one were $\alpha$ dystroglycan positive. Within mature fibers, $(C)$ the regenerating $n M H C$-positive fibers also stained positive for $\left(C^{\prime}\right) \alpha$-dystroglycan. Bar $=50 \mu m$.

single L276I mutations are asymptomatic, and patients homozygous for the L276I mutation often have a milder clinical course than most other patients with LGMD2I [24]. However, in patients who are compound heterozygous for the L276I mutation, the level of glycosylated, and thus functional, $\alpha$-dystroglycan is further reduced. The glycosylations are vital for interaction with the ECM protein laminin $\alpha 2$ (merosin) thus maintaining sarcolemmal stability [25]. All the patients who were compound heterozygous for the L276I mutation in our study are wheelchair users, and are severely affected by the disorder. Patients who are compound heterozygous for L276I/P462S and L276I/ Y307N have previously been described as having a more severe clinical presentation than patients homozygous for L276I [26,27], suggesting that, in most patients who are compound heterozygous for the L276I mutation, the other allele carries a mutation that renders FKRP even more dysfunctional than the homozygous L276I mutation itself.

Internally nucleated fibers are generally regarded as a marker for regenerating fibers after injury and muscular dystrophies, although there are rare exceptions to this (for example, centronuclear muscular dystrophy caused by impaired cohesion of the centrosome) $[28,29]$. In our study, we found that the number of internally nucleated fibers alone yielded very little information about the rate of regeneration, which probably relates to the fact that internally nucleated fibers can remain located in the cytoplasm, thus obscuring any difference in the rate of regeneration. This may explain why we did not find any significant relationship between internal nuclei and clinical severity, as expressed by the force in the muscle that we examined histologically. The absence of any trend towards change in internal nuclei throughout the age span in patients with the L276I mutation could be due 


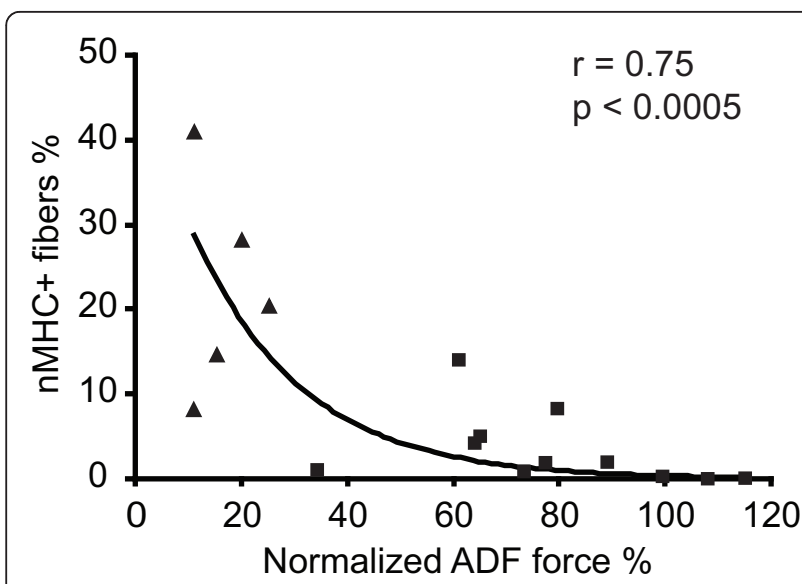

Figure $\mathbf{5}$ Regeneration versus muscle strength. There wass a significant relationship between regeneration in terms of number of neonatal myosin heavy chain ( $\mathrm{nMHC}$ )-positive fibers as a function of ankle dorsiflexion (ADF) strength in patients with LGMD2I, reflecting greater need for muscle regeneration in patients who have lost muscle strength through muscle wasting (squares = patients homozygous for L276l; triangles = patients compound heterozygous for L276I).

to several reasons, including differences in physical activity, fairly constant degeneration during the progression of the disorder, too few patients over a large age span, or the level of regeneration not being limited by available satellite cells. This is in contrast to what others have found in healthy humans; however, those findings were based on an age-dependent decline in number of satellite cells and not on actual regeneration [30]. Immunostaining for $\mathrm{nMHC}$ is useful in assessing ongoing regeneration in dystrophic muscle [31-33]. This $\mathrm{MHC}$ isoform has a peak expression within 10 days in regenerating fibers, thus providing a more precise temporal picture of the ongoing regeneration [34]. Although nMHC has been shown previously to be present in atrophic fibers (for example, in spinal muscular atrophy due to denervation related lack of muscle maturation [35]), we did not observe any upregulation of $\mathrm{nMHC}$ in atrophic fibers. Other markers for recent regeneration exist, but no other study has identified a better marker than $\mathrm{nMHC}$ for recent regeneration [36]. By adding utrophin staining, which is present in varying degrees on regenerating fibers, but is strongly upregulated in newly formed fibers, it is possible to see what the preferred method of regeneration is: repair of fibers and/or production of new fibers [20]. An increase in nMHCpositive fibers suggests that regeneration is progressing at a significantly higher pace in the L276I compound heterozygous patients, and that regeneration in these patients is mainly repair of degenerating fibers (nMHC +/utrophin-) rather than development of new myofibers (nMHC+/utrophin + ). We suggest that the increased level of $\mathrm{nMHC}$ reflects an increased rate of degeneration in severely affected patients, whereas patients with a milder phenotype apparently have little need for a high level of regeneration, because the resistance to structural stress is higher in the milder than in more severe form of LGMD2I, in which the fibers are more prone to degenerate. However, there is a possibility that delayed maturation is involved in the increased nMHC expression; that is, that the $\mathrm{nMHC}$ persists for longer than normal [37].

MyoD was not found in any of the muscles in this study. Presumably because of its short-lived action $(<48 \mathrm{hrs})$ it is difficult to detect MyoD by immunostaining of muscle tissue [38]. By contrast, myogenin is expressed up to a week after differentiation of the satellite cells is initiated [39]. The fact that we found myogenin-positive nuclei in the tissue of compound heterozygous patients is consistent with the increased number of $\mathrm{nMHC}+/$ utrophin-myofibers, and can be interpreted as a constant need for myofiber repair, that is, a higher rate of regeneration. However, whether this ultimately leads to exhaustion of the satellite-cell pool in LGMD2I and contributes to the worsening of the disease remains to be shown.

The trend towards a decrease in nMHC-positive fibers with age in L276I homozygous patients may reflect an agedependent rather than disorder-dependent decline in rate of regeneration. This seems inconsistent with the fact that we were unable to find a relationship between internal nuclei and age. However, activation of the satellite cells, the subsequent differentiation of the myoblasts leading to internal nuclei and the actual repair, although linked, are separate events, with the latter heavily dependent on protein synthesis (nMHC and other proteins), and age may affect these events differently. In patients who were compound heterozygous for the L276I mutation, the level of regeneration declined not only with age, but also with duration of illness. This implies that muscle repair decreases with time after onset, whereas muscle degeneration continues unabated, leading to progressive muscle wasting.

Recent advances in research of DMD therapy and research on sarcopenia have identified new opportunities for treating a wider field of muscular dystrophies by boosting the regenerative response, that is, by stimulating satellite-cell activation [40]. Knowledge of the existing regenerative response in LGMD2I may prove valuable, as differentiated regeneration may require different approaches for boosting the regenerative response to avoid adverse side effects, such as depleting the satellite-cell pool.

\section{Conclusions}

In this study, we found that although patients with LGMD2I who were homozygous for the L276I mutation appeared to have the same level of regeneration as 
patients who were compound heterozygous for this mutation, as determined by level of internally nucleated fibers, the level of early regeneration, as judged by the level of neonatal myosin heavy chain, was significantly higher in the compound heterozygous patients. These results yield a more accurate picture of the ongoing degeneration-regeneration cycle.

\section{Abbreviations}

a-DG: a-dystroglycan; FCS: fetal calf serum; FKRP: fukutin-related protein; LGMD2I: limb-girdle muscular dystrophy type 2l; L276I +/+: homozygous for the L276I mutation, L276I c.h.: compound heterozygous for the FKRP L276I mutation; nMHC: neonatal myosin heavy chain; PBS: phosphate-buffered saline.

\section{Acknowledgements}

We thank Eva Rahtkens, Danuta Olsen and Christina Andersen for excellent technical support. TK is supported financially by the Danish Medical Research Council, the Lundbeck Foundation, the Novo Nordisk Foundation and the AP Møller Foundation. JV is supported financially by the Sara and Ludvig Elssas Foundation.

\section{Author details}

'Neuromuscular Research Unit, Department of Neurology Rigshospitalet, University of Copenhagen, Denmark. ${ }^{2}$ Department of Clinical Genetics, Rigshospitalet, University of Copenhagen, Denmark.

\section{Authors' contributions}

TOK conceived, designed the study and carried out the histology/ immunohistology procedures as well data analysis and drafted the manuscript. SH carried out immunohistology and counted fibers, and performed the subsequent data analysis. MLS carried out force measurements on the patients. MS determined the mutations of the patients. JV provided biopsy material and helped to draft the manuscript. All authors read and approved the final manuscript.

\section{Competing interests}

The authors declare that they have no competing interests.

Received: 3 May 2011 Accepted: 5 October 2011

Published: 5 October 2011

\section{References}

1. Brockington M, Yuva Y, Prandini P, Brown SC, Torelli S, Benson MA, Herrmann R, Anderson LV, Bashir R, Burgunder JM, et al: Mutations in the fukutin-related protein gene (FKRP) identify limb girdle muscular dystrophy $2 \mathrm{l}$ as a milder allelic variant of congenital muscular dystrophy MDC1C. Hum Mol Genet 2001, 10:2851-2859.

2. Brockington M, Blake DJ, Prandini P, Brown SC, Torelli S, Benson MA

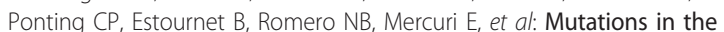
fukutin-related protein gene (FKRP) cause a form of congenital muscular dystrophy with secondary laminin alpha2 deficiency and abnormal glycosylation of alpha-dystroglycan. Am J Hum Genet 2001, 69:1198-1209.

3. Ibraghimov-Beskrovnaya O, Ervasti JM, Leveille CJ, Slaughter CA, Sernett SW, Campbell KP: Primary structure of dystrophin-associated glycoproteins linking dystrophin to the extracellular matrix. Nature 1992, 20;355:696-702.

4. Esapa CT, Mcllhinney RA, Blake DJ: Fukutin-related protein mutations that cause congenital muscular dystrophy result in ER-retention of the mutant protein in cultured cells. Hum Mol Genet 2005, 14:295-305.

5. Torelli S, Brown SC, Brockington M, Dolatshad NF, Jimenez C, Skordis $L$, Feng LH, Merlini L, Jones DH, Romero N, et al: Sub-cellular localisation of fukutin related protein in different cell lines and in the muscle of patients with MDC1C and LGMD2I. Neuromuscul Disord 2005, 15:836-843.

6. Sveen ML, Schwartz M, Vissing J: High prevalence and phenotypegenotype correlations of limb girdle muscular dystrophy type 21 in Denmark. Ann Neurol 2006, 59:808-815.
7. Sveen ML, Schwartz M, Vissing J: High prevalence and phenotypegenotype correlations of limb girdle muscular dystrophy type 21 in Denmark. Ann Neurol 2006, 59:808-815.

8. Schwartz M, Hertz JM, Sveen ML, Vissing J: LGMD2I presenting with a characteristic Duchenne or Becker muscular dystrophy phenotype. Neurology 2005, 64:1635-1637.

9. Brockington M, Yuva Y, Prandini P, Brown SC, Torelli S, Benson MA, Herrmann R, Anderson LV, Bashir R, Burgunder JM, et al: Mutations in the fukutin-related protein gene (FKRP) identify limb girdle muscular dystrophy 21 as a milder allelic variant of congenital muscular dystrophy MDC1C. Hum Mol Genet 2001, 10:2851-2859.

10. Sveen ML, Schwartz M, Vissing J: High prevalence and phenotypegenotype correlations of limb girdle muscular dystrophy type 21 in Denmark. Ann Neurol 2006, 59:808-815.

11. Wroblewski R, Edstrom L, Mair WG: Five different types of centrally nucleated muscle fibres in man: elemental composition and morphological criteria. A study of myopathies, fetal tissue and muscle spindle. J Submicrosc Cytol 1982, 14:377-387.

12. Sartore $S$, Gorza L, Schiaffino S: Fetal myosin heavy chains in regenerating muscle. Nature 1982, 298:294-296.

13. Grounds MD, Garrett KL, Lai MC, Wright WE, Beilharz MW: Identification of skeletal muscle precursor cells in vivo by use of MyoD1 and myogenin probes. Cell Tissue Res 1992, 267:99-104.

14. Marsh DR, Criswell DS, Carson JA, Booth FW: Myogenic regulatory factors during regeneration of skeletal muscle in young, adult, and old rats. $J$ Appl Physiol 1997, 83:1270-1275

15. Jejurikar SS, Kuzon WM Jr: Satellite cell depletion in degenerative skeletal muscle. Apoptosis 2003, 8:573-578.

16. Sveen ML, Schwartz M, Vissing J: High prevalence and phenotypegenotype correlations of limb girdle muscular dystrophy type 21 in Denmark. Ann Neurol 2006, 59:808-815.

17. Fanin $M$, Nascimbeni AC, Fulizio $L$, Trevisan CP, Meznaric-Petrusa M, Angelini C: Loss of calpain-3 autocatalytic activity in LGMD2A patients with normal protein expression. Am J Pathol 2003, 163:1929-1936.

18. Ponten $\mathrm{E}$, Friden J: Immobilization of the rabbit tibialis anterior muscle in a lengthened position causes addition of sarcomeres in series and extracellular matrix proliferation. J Biomech 2008, 41:1801-1804.

19. Cohn RD, Henry MD, Michele DE, Barresi R, Saito F, Moore SA, Flanagan JD, Skwarchuk MW, Robbins ME, Mendell JR, et al: Disruption of DAG1 in differentiated skeletal muscle reveals a role for dystroglycan in muscle regeneration. Cell 2002, 110:639-648.

20. Helliwell TR, Man NT, Morris GE, Davies KE: The dystrophin-related protein, utrophin, is expressed on the sarcolemma of regenerating human skeletal muscle fibres in dystrophies and inflammatory myopathies. Neuromuscul Disord 1992, 2:177-184.

21. Hodges BL, Hayashi YK, Nonaka I, Wang W, Arahata K, Kaufman SJ: Altered expression of the alpha7beta 1 integrin in human and murine muscular dystrophies. J Cell Sci 1997, 110:2873-2881.

22. Blake DJ, Tinsley JM, Davies KE: Utrophin: a structural and functional comparison to dystrophin. Brain Pathol 1996, 6:37-47.

23. Nguyen TM, Ellis JM, Love DR, Davies KE, Gatter KC, Dickson G, Morris GE: Localization of the DMDL gene-encoded dystrophin-related protein using a panel of nineteen monoclonal antibodies: presence at neuromuscular junctions, in the sarcolemma of dystrophic skeletal muscle, in vascular and other smooth muscles, and in proliferating brain cell lines. J Cell Biol 1991, 115:1695-1700.

24. de Paula F, Vieira N, Starling A, Yamamoto LU, Lima B, de Cassia PR, Vainzof M, Nigro V, Zatz M: Asymptomatic carriers for homozygous novel mutations in the FKRP gene: the other end of the spectrum. Eur I Hum Genet 2003, 11:923-930

25. Brockington M, Blake DJ, Prandini P, Brown SC, Torelli S, Benson MA, Ponting $C P$, Estournet $B$, Romero NB, Mercuri $E$, et al: Mutations in the fukutin-related protein gene (FKRP) cause a form of congenital muscular dystrophy with secondary laminin alpha2 deficiency and abnormal glycosylation of alpha-dystroglycan. Am J Hum Genet 2001, 69:1198-1209.

26. Schwartz M, Hertz JM, Sveen ML, Vissing J: LGMD2I presenting with a characteristic Duchenne or Becker muscular dystrophy phenotype. Neurology 2005, 64:1635-1637.

27. Mercuri E, Brockington M, Straub V, Quijano-Roy S, Yuva Y, Herrmann R, Brown SC, Torelli S, Dubowitz V, Blake DJ, et al: Phenotypic spectrum 
associated with mutations in the fukutin-related protein gene. Ann Neurol 2003, 53:537-542.

28. Paulsen $G$, Egner IM, Drange M, Langberg H, Benestad HB, Fjeld JG, Hallen J, Raastad T: A COX-2 inhibitor reduces muscle soreness, but does not influence recovery and adaptation after eccentric exercise. Scand J Med Sci Sports 2010, 20:e195-e207.

29. Dubowitz V, Sewry CA: Muscle Biopsy - A practical Approach , 32007.

30. Corbu A, Scaramozza A, Badiali-DeGiorgi L, Tarantino L, Papa V, Rinaldi R, D'Alessandro R, Zavatta M, Laus M, Lattanzi G, et al: Satellite cell characterization from aging human muscle. Neurol Res 2010, 32:63-72.

31. Fitzsimons RB, Hoh JF: Embryonic and foetal myosins in human skeletal muscle. The presence of foetal myosins in duchenne muscular dystrophy and infantile spinal muscular atrophy. J Neurol Sci 1981, 52:367-384.

32. Schiaffino S, Gorza L, Dones I, Cornelio F, Sartore S: Fetal myosin immunoreactivity in human dystrophic muscle. Muscle Nerve 1986, 9:51-58.

33. Winter A, Bornemann A: NCAM, vimentin and neonatal myosin heavy chain expression in human muscle diseases. Neuropathol Appl Neurobiol 1999, 25:417-424.

34. Sartore S, Gorza L, Schiaffino S: Fetal myosin heavy chains in regenerating muscle. Nature 1982, 298:294-296.

35. Biral D, Scarpini E, Angelini C, Salviati G, Margreth A: Myosin heavy chain composition of muscle fibers in spinal muscular atrophy. Muscle Nerve 1989, 12:43-51.

36. Winter A, Bornemann A: NCAM, vimentin and neonatal myosin heavy chain expression in human muscle diseases. Neuropathol Appl Neurobiol 1999, 25:417-424.

37. Schiaffino S, Gorza L, Dones I, Cornelio F, Sartore S: Fetal myosin immunoreactivity in human dystrophic muscle. Muscle Nerve 1986, 9:51-58.

38. Grounds MD, Garrett KL, Lai MC, Wright WE, Beilharz MW: Identification of skeletal muscle precursor cells in vivo by use of MyoD1 and myogenin probes. Cell Tissue Res 1992, 267:99-104.

39. Marsh DR, Criswell DS, Carson JA, Booth FW: Myogenic regulatory factors during regeneration of skeletal muscle in young, adult, and old rats. J Appl Physiol 1997, 83:1270-1275.

40. Voisin V, Sebrie C, Matecki S, Yu H, Gillet B, Ramonatxo M, Israel M, De la PS: L-arginine improves dystrophic phenotype in $\mathrm{mdx}$ mice. Neurobiol Dis 2005, 20:123-130.

doi:10.1186/2044-5040-1-31

Cite this article as: Krag et al:: Level of muscle regeneration in limbgirdle muscular dystrophy type 21 relates to genotype and clinical severity. Skeletal Muscle 2011 1:31.

\section{Submit your next manuscript to BioMed Central and take full advantage of:}

- Convenient online submission

- Thorough peer review

- No space constraints or color figure charges

- Immediate publication on acceptance

- Inclusion in PubMed, CAS, Scopus and Google Scholar

- Research which is freely available for redistribution

Submit your manuscript at www.biomedcentral.com/submit
C Biomed Central 